\title{
An ANN-Based Synthesis Model for Parallel Coupled Microstrip Lines with Floating Ground-Plane Conductor and Its Applications
}

\author{
Yuan Cao, ${ }^{1,2}$ Zhongbao Wang, $^{1,3}$ and Shaojun Fang ${ }^{1}$ \\ ${ }^{1}$ School of Information Science and Technology, Dalian Maritime University, Dalian, Liaoning 116026, China \\ ${ }^{2}$ School of Electrical and Control Engineering, Liaoning Technical University, Huludao, Liaoning 125105, China \\ ${ }^{3}$ School of Electronic Engineering, Beijing University of Posts and Telecommunications, Beijing 100876, China \\ Correspondence should be addressed to Zhongbao Wang; wzbdlmu@foxmail.com
}

Received 15 October 2015; Revised 2 February 2016; Accepted 11 February 2016

Academic Editor: Diego Masotti

Copyright (c) 2016 Yuan Cao et al. This is an open access article distributed under the Creative Commons Attribution License, which permits unrestricted use, distribution, and reproduction in any medium, provided the original work is properly cited.

\begin{abstract}
To directly obtain physical dimensions of parallel coupled microstrip lines with a floating ground-plane conductor (PCMLFGPC), an accurate synthesis model based on an artificial neural network (ANN) is proposed. The synthesis model is validated by using the conformal mapping technique (CMT) analysis contours. Using the synthesis model and the CMT analysis, the PCMLFGPC having equal even- and odd-mode phase velocities can be obtained by adjusting the width of the floating ground-plane conductor. Applying the method, a $7 \mathrm{~dB}$ coupler with the measured isolation better than $27 \mathrm{~dB}$ across a wide bandwidth (more than $120 \%$ ), a $90^{\circ}$ Schiffman phase shifter with phase deviation $\pm 2.5^{\circ}$ and return loss more than $17.5 \mathrm{~dB}$ covering $63.4 \%$ bandwidth, and a bandpass filter with completely eliminated second-order spurious band are implemented. The performances of the current designs are superior to those of the previous components configured with the PCMLFGPC.
\end{abstract}

\section{Introduction}

The parallel coupled microstrip lines (PCML) have been widely used in microwave circuits [1] such as directional couplers $[2,3]$, phase shifters $[4]$, and filters $[5,6]$. For the traditional PCML, the coupling factor is mainly dependent on the spacing between the two coupled microstrip lines and the dielectric constant of the used substrate. When using the general printed circuit board (PCB) fabrication technology, it is difficult to realize a tight coupling (i.e., $<10 \mathrm{~dB}$ ) as very narrow spacing is required [1]. Actually, PCML with the tight coupling are required in wideband applications such as $3 \mathrm{~dB}$ coupler for wideband circularly polarized patch antennas [7], Schiffman phase shifters [8], and ultra-wideband filters [9]. In order to increase the level of coupling, a groundplane aperture was introduced used to design directional couplers in [10] and bandpass filters in [11, 12]. The groundplane aperture was also adopted to tune even- and oddmode phase velocities for high isolation couplers $[13,14]$ and parallel coupled microstrip bandpass filters with spurious band suppression [15]. In [16], a floating conductor strip was firstly inserted in the aperture etched in the ground plane under the coupled lines to further enhance the level of coupling and equalize even- and odd-mode phase velocities for wideband bandpass filter applications. Then, wideband Schiffman phase shifters and couplers were also designed by using the parallel coupled microstrip lines with floating ground-plane conductors (PCMLFGPC) $[17,18]$. The analysis formulas of the PCMLFGPC were derived by Abbosh [19] with the help of the conformal mapping technique (CMT) to calculate the even- and odd-mode impedance. However, complicated CMT analysis formulas need strong background knowledge to be understood and used. Moreover, an iteration technique of applying the analysis formulas to calculate physical dimensions of the PCMLFGPC is required. Therefore, with increasing popularity of the PCMLFGPC for the design of microwave circuits, it is highly desirable to have an accurate 


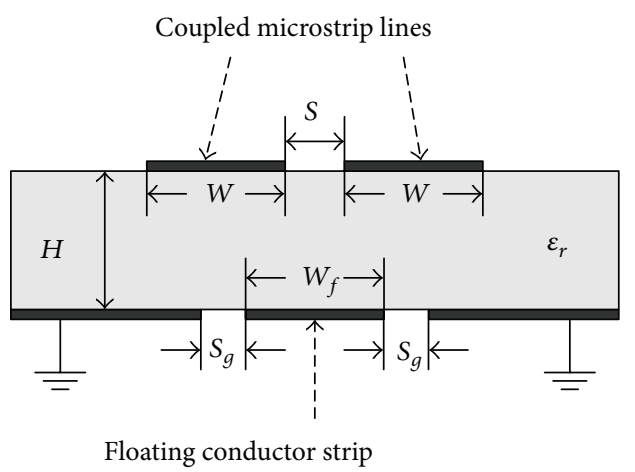

FIGURE 1: Cross-sectional view of parallel coupled microstrip lines with a floating ground-plane conductor.

synthesis method for directly obtaining physical dimensions of the PCMLFGPC.

Recently, artificial neural networks (ANNs) have been used to synthesize microwave transmission lines [20] such as coplanar strip lines [21], coplanar waveguides [22], and impedance-varying microstrip lines [23]. However, the input/ output configuration and the required neuron number of the ANN for a given modeling task need to be determined in the practical application. Furthermore, to the best of our knowledge, there is no synthesis model for the PCMLFGPC.

In this paper, an ANN-based synthesis model for the PCMLFGPC is proposed to directly obtain physical dimensions of the structure. A high isolation coupler, a wideband Schiffman phase shifter, and a bandpass filter with secondorder spurious band suppression are designed by using the synthesis model, which is validated by comparing their results with the electromagnetic simulation and measurement.

\section{ANN-Based Synthesis Model}

Figure 1 shows the cross section view of a PCMLFGPC. $W$ represents the width of the parallel coupled microstrip lines; $S$ represents the spacing between parallel coupled microstrip lines. The dielectric substrate has thickness of $H$ with relative dielectric permittivity $\varepsilon_{r}$. The width of the floating conductor strip is $W_{f}$. The width of slots between the floating conductor strip and the ground plane is $S_{g}$. The even- and oddmode characteristic impedance $\left(Z_{0 e}\right.$ and $\left.Z_{0 o}\right)$ and effective dielectric constant $\left(\varepsilon_{e e}\right.$ and $\left.\varepsilon_{e o}\right)$ depend on the dimensions of the coupled structure. The complicated relation between them is a group of high-dimensional and highly nonlinear equations [19], which cannot be easily solved to obtain physical dimensions of the PCMLFGPC with respect to the required electrical parameters. In order to overcome this problem, ANN will be used in this paper.

As a fast and flexible vehicle for microwave modeling, ANNs have been developed for many years [24]. Among a variety of ANNs, multilayer perceptron (MLP) is most suitable to model these high-dimensional and highly nonlinear problems. Thus, an MLP neural network is adopted in this study, which consists of three types of layers: an input layer, an output layer, and one or more hidden layers, as shown in Figure 2. More details about the MLP can be found in [24, 25].

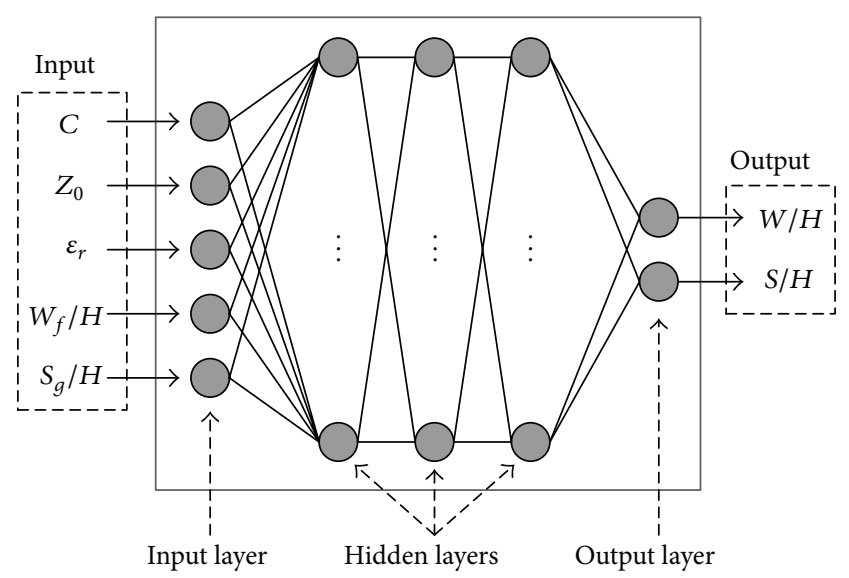

FIgURE 2: ANN-based synthesis model of the PCMLFGPC.

An ANN used to synthesize microwave transmission lines can be named an "inverse model," in which the model inputs are electrical parameters and the outputs are geometric parameters [26]. It is used to find the geometric parameters for the given values of the electrical parameters. Unlike the analysis model, in which the input-to-output mapping is usually a one-to-one mapping, the inverse model often encounters the problem of multiple solutions [26]. This problem also causes difficulties during training because the same input values to the inverse model will lead to different values of the output. As a result, the neural network inverse model cannot be trained accurately [26]. To solve this problem, some geometric parameters are used as input values in our synthesis model to ensure a unique value of the output. Figure 2 illustrates the proposed synthesis model. The synthesis model can be used to calculate the coupledlines width $W$ and coupled-lines spacing $S$ for the required coupling factor $C$ and port impedance $Z_{0}$ with the given substrate $\left(\varepsilon_{r}\right.$ and $\left.H\right)$ by choosing the appropriate floating conductor width $W_{f}$ and ground-plane slot width $S_{g}$.

The ANN-based model is a kind of black box model, whose accuracy depends on the training data sets and network configuration [27]. To obtain the training and test data sets, CMT analysis formulas in [19] are used to generate data sets for $2 \leq \varepsilon_{r} \leq 12,0.5 \leq W / H \leq 5,0.05 \leq S / H \leq 1$, $1 \leq W_{f} / H \leq 10,0.5 \leq S_{g} / H \leq 1,0.30 \leq C \leq 0.75$, and $30 \Omega \leq Z_{0} \leq 150 \Omega$. In this study, out of the 5000 data sets generated, 4000 are used for training, and the rest are used to test the trained ANNs. For the network configuration, a three-hidden-layered network is found that the target of high accuracy can be achieved. The suitable network configuration for the synthesis model is $5 \times 20 \times 40 \times 16 \times 2$. The numbers of neurons are 5, 20, 40, 16, and 2 for the input layer, the first, second, and third hidden layers, and the output layer, respectively. The activation function of neurons, normalization of training data, and the training algorithm are chosen the same as [28]. The maximum allowable number of training epochs is 400 . At last, the average relative error between the ANN predicted value and the CMT analysis result is less than $0.8 \%$, and maximal relative error [28] is less than $2.9 \%$. 


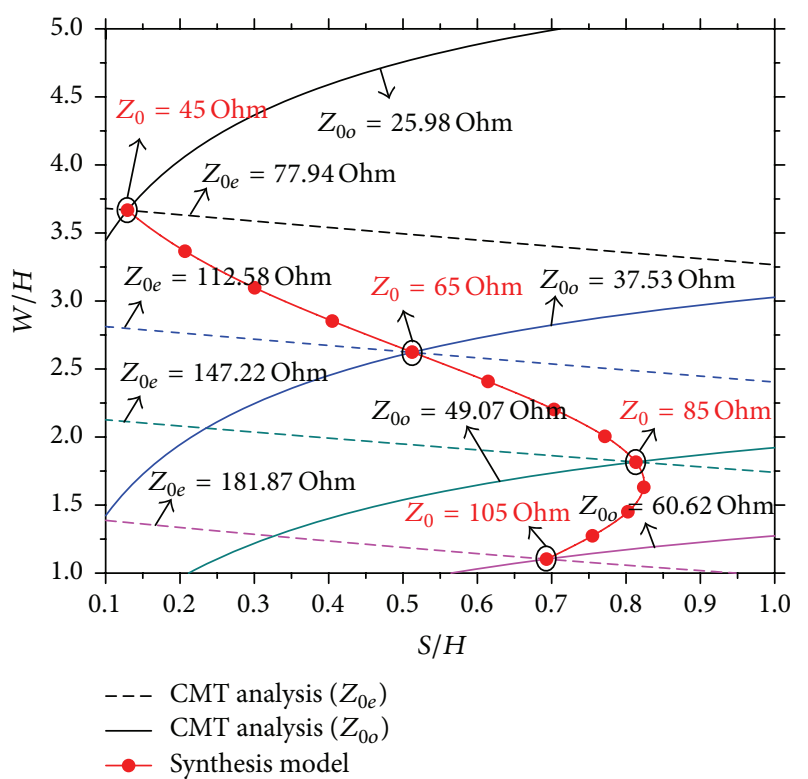

FIGURE 3: Comparison of results obtained from ANN-based synthesis model and CMT analysis contours for the PCMLFGPC with $\varepsilon_{r}=3.5, W_{f} / H=3.5, S_{g} / H=1$, and $C=0.5$.

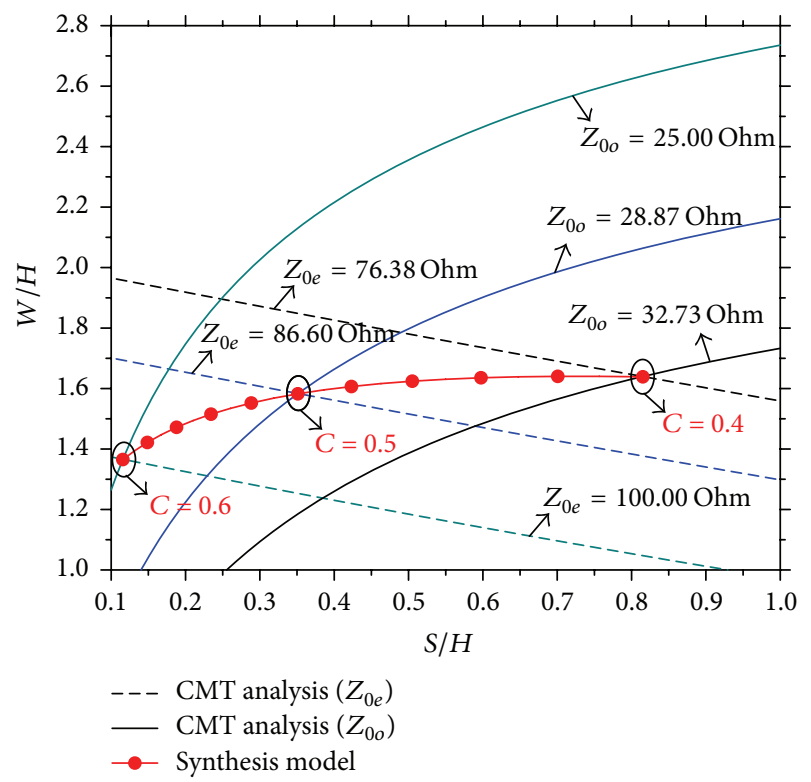

FIGURE 4: Comparison of results obtained from ANN-based synthesis model and CMT analysis contours for the PCMLFGPC with $\varepsilon_{r}=9.8, W_{f} / H=3, S_{g} / H=0.5$, and $Z_{0}=50 \mathrm{Ohm}$.

\section{Validation of Synthesis Model}

In order to validate the proposed synthesis model for the PCMLFGPC, the results obtained from the proposed model compared with the CMT analysis contours are shown graphically in Figures 3 and 4.

Figure 3 plots the ratios of geometrical dimensions $W / H$ versus the $S / H$ for various values of port impedance $Z_{0}$ with the given substrate material, floating conductor width, ground-plane slot width, and equal coupling factor. Figure 4 plots the ratios of geometrical dimensions $W / H$ versus the $S / H$ for various values of coupling factor $C$ with the given substrate material, floating conductor width, ground-plane slot width, and equal port impedance $Z_{0}$. Excellent agreements between the results of the CMT analysis and the ANNbased synthesis are obtained, validating the proposed method for the synthesis of the PCMLFGPC.

\section{Applications of Synthesis Model}

In order to further validate the proposed model for the synthesis of the PCMLFGPC, a high isolation coupler, a wideband Schiffman phase shifter, and a bandpass filter are designed, simulated, fabricated, and measured in this section.

4.1. Design of a $7 \mathrm{~dB}$ Directional Coupler. As the first example, a $1.5 \mathrm{GHz}$ coupler with coupling factor $C=0.4467$ (or $7 \mathrm{~dB}$ ) and port impedance $Z_{0}=50 \mathrm{Ohm}$ is designed by using the proposed synthesis model. The used substrate has relative dielectric permittivity $\varepsilon_{r}=3.5$ and thickness $H=1.5 \mathrm{~mm}$, which also is used to implement the phase shifter and bandpass filter in Sections 4.2 and 4.3. In the design of the $7 \mathrm{~dB}$ coupler, the ground-plane slot width is selected as $S_{g}=$ $0.75 \mathrm{~mm}$ (i.e., $S_{g} / H=0.5$ ). Then, the outputs of the synthesis model of $W / H$ and $S / H$ (i.e., coupled-lines width $W$ and coupled-lines spacing $S$ ) are only varied with $W_{f} / H$. To realize a higher isolation, the floating conductor width $W_{f}$ is adjusted to accomplish equal even- and odd-mode effective dielectric constant. Last, the floating conductor width is determined to be $W_{f}=6.9 \mathrm{~mm}$ (i.e., $W_{f} / H=4.6$ ). Meanwhile, the calculated coupled-lines width $W$ and coupledlines spacing $S$ using the synthesis model, respectively, are $5.0 \mathrm{~mm}$ and $0.5 \mathrm{~mm}$ with $\varepsilon_{e e}=\varepsilon_{e o}=2.58$. Concerning the coupled-line length $L$, it is chosen to be a quarter of the effective wavelength at $1.5 \mathrm{GHz}$. Then, the coupled-line length $L$ is calculated to be $31.1 \mathrm{~mm}$. Using calculated geometrical dimensions and HFSS electromagnetic simulator, the simulated strongest coupling is at $1.433 \mathrm{GHz}$ with $7.5 \mathrm{~dB}$ (greater than the required $7 \mathrm{~dB}$ ), which is mainly due to the radiation loss from the slots in the ground plane. The central operation frequency shift is likely due to the inaccuracies of the CMT analysis formulas [19]. However, the discrepancies can be corrected by slightly adjusting $W, S$, and $L$. Finally, the geometrical dimensions of the $7 \mathrm{~dB}$ coupler are found to be $W=4.7 \mathrm{~mm}, S=0.47 \mathrm{~mm}, L=30 \mathrm{~mm}, S_{g}=0.75 \mathrm{~mm}$, and $W_{f}=6.9 \mathrm{~mm}$.

Figure 5 shows the top and bottom views of the fabricated coupler with overall circuit size of $140 \mathrm{~mm} \times 50 \mathrm{~mm}$. $S$ parameter measurements were performed by using an Agilent N5230A network analyzer and compared with HFSS simulations, as shown in Figures 6 and 7. The measured value of coupling is $7.3 \mathrm{~dB}$ at the central operation frequency $1.5 \mathrm{GHz}$. The $0.3 \mathrm{~dB}$ discrepancy probably arises from SMA connectors, which were not included in the simulation. It is worth mentioning that the measured isolation is better than $27 \mathrm{~dB}$, return loss is better than $20 \mathrm{~dB}$, and output phase difference between the coupled and through ports is $90^{\circ} \pm 1^{\circ}$ from 0.6 to $2.4 \mathrm{GHz}$. 


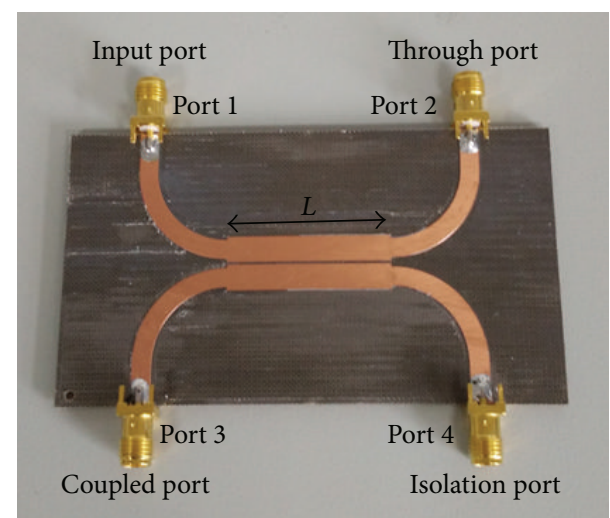

(a) Top view

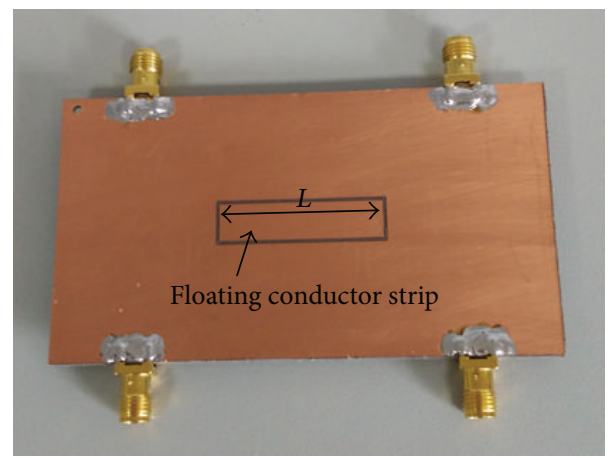

(b) Bottom view

FIGURE 5: Photograph of the fabricated $7 \mathrm{~dB}$ coupler configured with the PCMLFGPC.

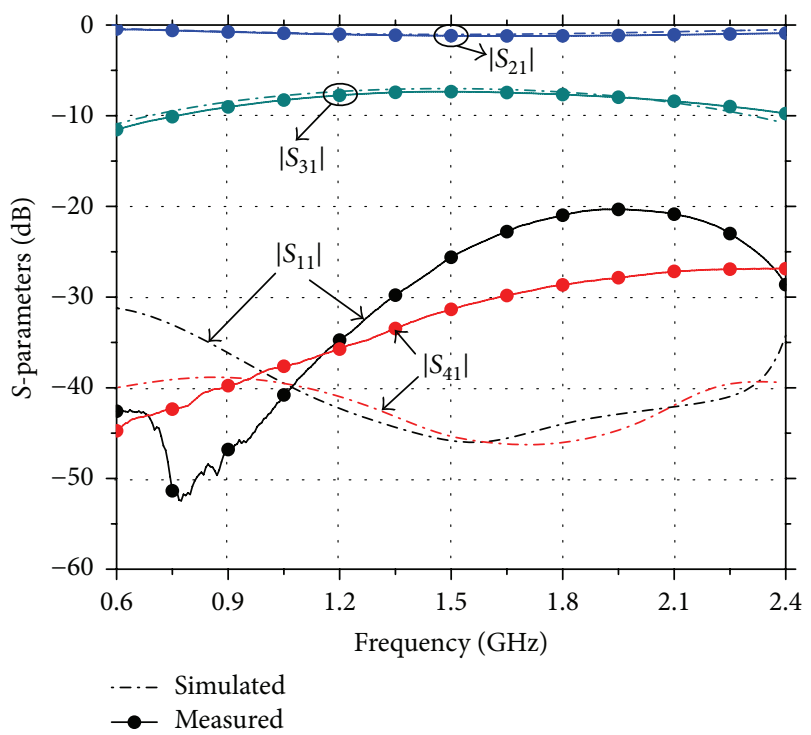

FIGURE 6: Simulated and measured $S$-parameters of the $7 \mathrm{~dB}$ coupler configured with the PCMLFGPC.

4.2. Design of a $90^{\circ}$ Schiffman Phase Shifter. In this second example, a $90^{\circ}$ Schiffman phase shifter configured with the PCMLFGPC is designed, which is previously presented by

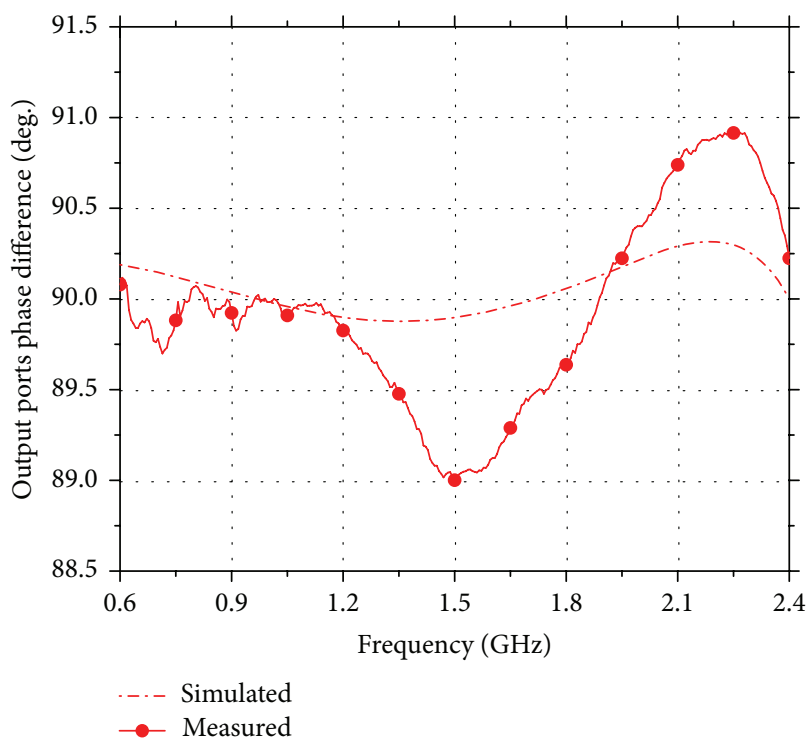

FIGURE 7: Simulated and measured output ports phase difference of the $7 \mathrm{~dB}$ coupler configured with the PCMLFGPC.

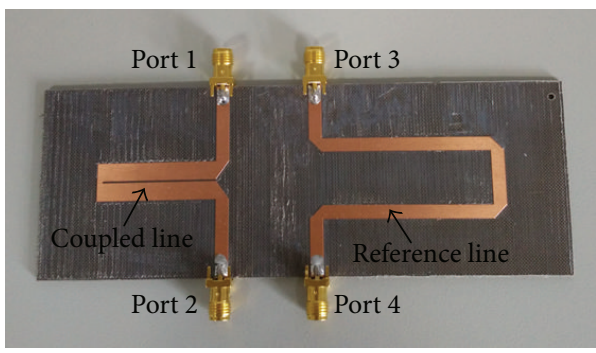

(a) Top view

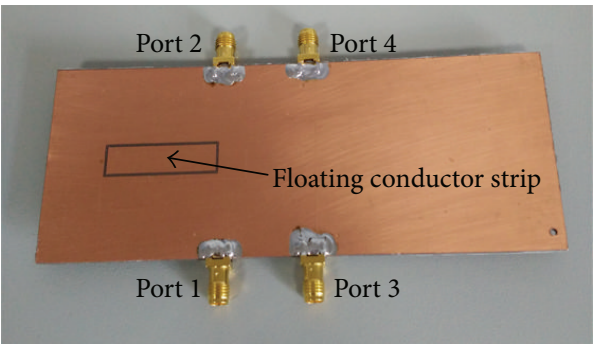

(b) Bottom view

FIGURE 8: Photograph of the fabricated $90^{\circ}$ Schiffman phase shifter configured with the PCMLFGPC.

Guo et al. [17]. Similar to [17], the coupling factor of coupledline section is chosen to be $7 \mathrm{~dB}$. For conciseness, geometrical dimensions of the PCMLFGPC in the phase shifter are the same as that in the foregoing coupler. Figure 8 gives top and bottom views of the fabricated $90^{\circ}$ Schiffman phase shifter. The overall circuit size is $190 \mathrm{~mm} \times 50 \mathrm{~mm}$.

Figure 9 gives the simulated and measured $S$-parameter of the phase shifter. The measured insertion loss is lower than $0.7 \mathrm{~dB}$ and return loss is better than $17.5 \mathrm{~dB}$ from 0.6 to $2.4 \mathrm{GHz}$. Measured bandwidth for phase derivation within $\pm 2.5^{\circ}$ is about $63.4 \%$ from 1.086 to $2.094 \mathrm{GHz}$, and that for 


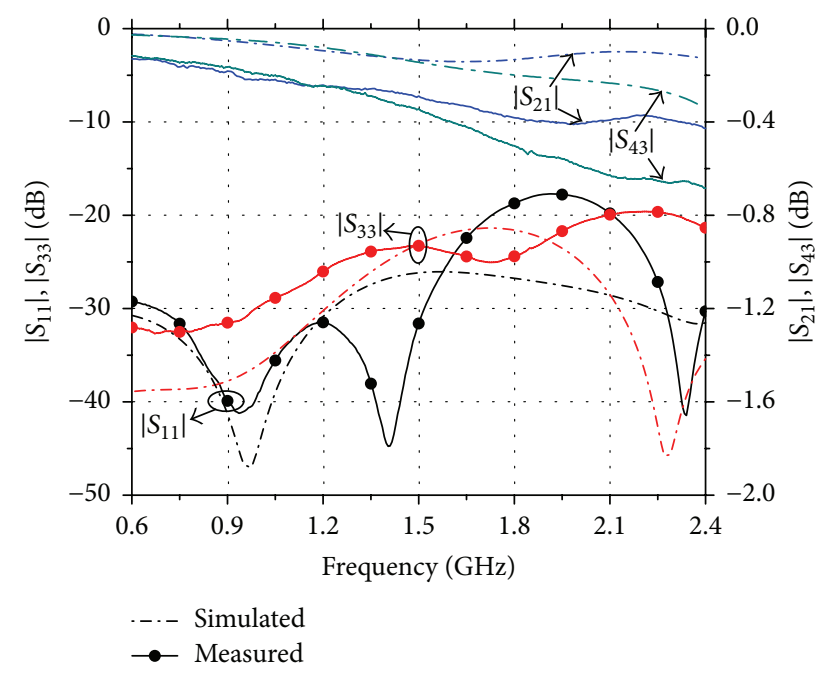

Figure 9: Simulated and measured $S$-parameters of the $90^{\circ}$ Schiffman phase shifter configured with the PCMLFGPC.

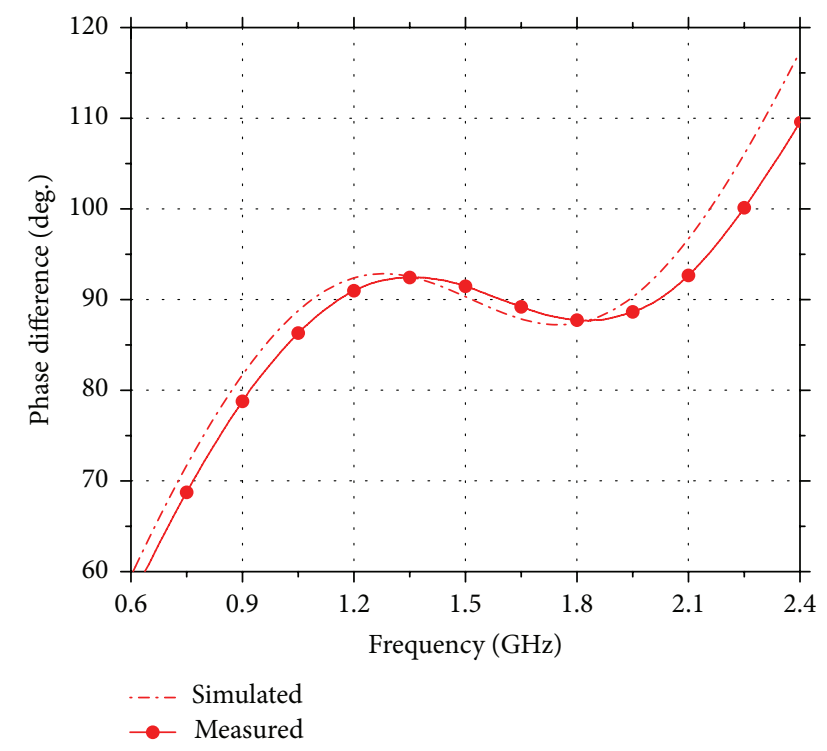

FIGURE 10: Simulated and measured phase difference responses of the $90^{\circ}$ Schiffman phase shifter configured with the PCMLFGPC.

$\pm 5^{\circ}$ is $71.5 \%$ ( 1.020 to $2.154 \mathrm{GHz}$ ), as shown in Figure 10. It is noted that the insertion loss, return loss, and bandwidth of the current design are all superior to that of the previously phase shifter in [17].

4.3. Design of a Chebyshev Bandpass Filter. In this last example, we design a $1.5 \mathrm{GHz}$ one-pole Chebyshev bandpass filter with a bandwidth of $30 \%$ and ripple of $0.5 \mathrm{~dB}$. Based on the filter theory [1], two identical coupled sections with $Z_{0 e}=$ 124.8 Ohm and $Z_{0 o}=42.7 \mathrm{Ohm}$ are calculated. Thus, the coupling factor and port impedance of the PCMLFGPC for the filter are $C=0.49$ (about $6.2 \mathrm{~dB}$ ) and $Z_{0}=73 \mathrm{Ohm}$, respectively. Similar to the foregoing examples, the groundplane slot width is also selected as $S_{g}=0.75 \mathrm{~mm}$ and the

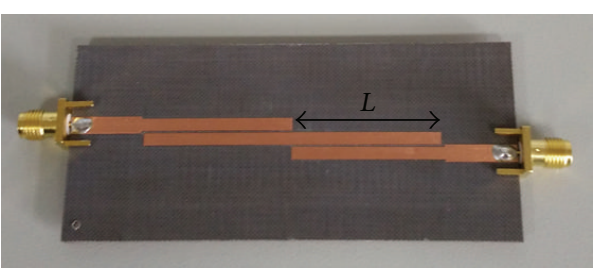

(a) Top view

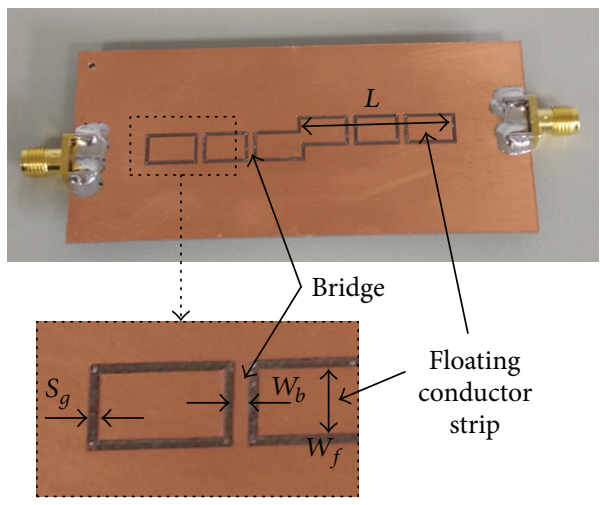

(b) Bottom view

FIgURE 11: Photograph of the fabricated bandpass filter configured with the PCMLFGPC.

floating conductor width $W_{f}$ is adjusted to obtain equal evenand odd-mode effective dielectric constant. In this case, the floating conductor width is determined to be $W_{f}=4.935 \mathrm{~mm}$ (i.e., $W_{f} / H=3.29$ ). Meanwhile, the calculated coupled-lines width $W$ and coupled-lines spacing $S$ using the synthesis model, respectively, are $2.66 \mathrm{~mm}$ and $0.50 \mathrm{~mm}$ with $\varepsilon_{e e}=$ $\varepsilon_{e o}=2.42$.

Similar to [16], the floating conductor is divided into three identical sections by using metal bridges to avoid the generation of undesired ground-plane slot modes, as shown in Figure 11 (the bottom view of the filter). The width of all the bridges is assigned equal to $1.0 \mathrm{~mm}$ and all the spacing between floating conductors and the ground plane is chosen to be $0.75 \mathrm{~mm}$. Finally, the geometrical dimensions of the bandpass filter are implemented to be $W=2.50 \mathrm{~mm}, S=$ $0.46 \mathrm{~mm}, L=30 \mathrm{~mm}, S_{g}=0.75 \mathrm{~mm}, W_{f}=4.935 \mathrm{~mm}$, and $W_{b}=1.0 \mathrm{~mm}$. Figure 12 gives the simulated and measured $S$-parameters of the bandpass filter configured with the PCMLFGPC. It is clearly observed that second-order spurious band of the bandpass filter is completely eliminated by using the PCMLFGPC synthesized with the proposed method.

\section{Conclusion}

In this paper, the ANN-based computer-aided design model for the synthesis of the PCMLFGPC has been presented. Using the synthesis model, the physical dimensions of the PCMLFGPC can be directly obtained. Furthermore, with the help of the CMT analysis, it is very easy to achieve equal even- and odd-mode phase velocities of the PCMLFGPC, which will improve the isolation of directional couplers, 


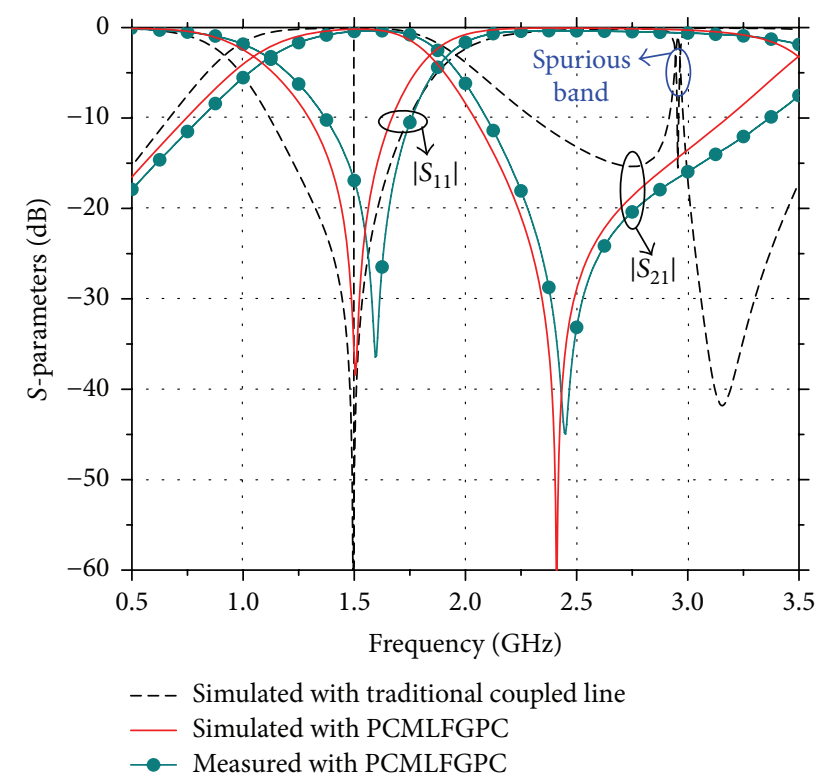

FIGURE 12: Comparison of the performances of bandpass filters configured with traditional parallel coupled microstrip lines or the PCMLFGPC.

increase the return loss of Schiffman phase shifters, and completely eliminate second-order spurious band of parallel coupled microstrip bandpass filters. The current method has been validated in practical applications with simulation and measurement.

\section{Competing Interests}

The authors declare that they have no competing interests.

\section{Acknowledgments}

This work was supported jointly by the National Natural Science Foundation of China (nos. 61401056 and 61571075), the Doctor Startup Foundation of Liaoning Province (no. 20141103), the Scientific Research Project of the Department of Education of Liaoning Province (no. L2013196), the China Postdoctoral Science Foundation (no. 2015M580070), and the Fundamental Research Funds for the Central Universities (no. 3132015212).

\section{References}

[1] R. K. Mongia, I. J. Bahl, P. Bhartia, and J. Hong, RF and Microwave Coupled-Line Circuits, Artech House, Norwood, Mass, USA, 2nd edition, 2007.

[2] I. Arnedo, I. Arregui, M. Chudzik et al., "Passive microwave component design using inverse scattering: theory and applications," International Journal of Antennas and Propagation, vol. 2013, Article ID 761278, 10 pages, 2013.

[3] Y. Wu, W. Sun, S.-W. Leung, Y. Diao, K.-H. Chan, and Y.-M. Siu, "Single-layer microstrip high-directivity coupled-line coupler with tight coupling," IEEE Transactions on Microwave Theory and Techniques, vol. 61, no. 2, pp. 746-753, 2013.
[4] Q. Liu, Y. Liu, J. Shen et al., "Wideband single-layer $90^{\circ}$ phase shifter using stepped impedance open stub and coupled-line with weak coupling," IEEE Microwave and Wireless Components Letters, vol. 24, no. 3, pp. 176-178, 2014.

[5] F. Xiao, M. Norgren, and S. He, "Quasi-TEM approach of coupled-microstrip lines and its application to the analysis of microstrip filters," International Journal of RF and Microwave Computer-Aided Engineering, vol. 22, no. 1, pp. 131-139, 2012.

[6] Y. Wu, S. Zhou, W. Zhang, M. Liao, and Y. Liu, "Coupledline dual-band bandpass filter with compact structure and wide stopband," Electronics Letters, vol. 50, no. 3, pp. 187-189, 2014.

[7] H. L. Chung, X. Qing, and Z. N. Chen, "A broadband circularly polarized stacked probe-fed patch antenna for UHF RFID applications," International Journal of Antennas and Propagation, vol. 2007, Article ID 76793, 8 pages, 2007.

[8] J. L. R. Quirarte and J. P. Starski, "Synthesis of Schiffman phase shifters," IEEE Transactions on Microwave Theory and Techniques, vol. 39, no. 11, pp. 1885-1889, 1991.

[9] W. Feng and W. Che, "Novel ultra-wideband bandpass filter using shorted coupled lines and transversal transmission line," IEEE Microwave and Wireless Components Letters, vol. 24, no. 3, pp. 548-550, 2014.

[10] M. Aikawa, "Microstrip line directional coupler with tight coupling and high directivity," IECE Transactions, vol. 60, no. 4, pp. 253-259, 1977.

[11] L. Zhu, H. Bu, and K. Wu, "Broadband and compact multi-pole microstrip bandpass filters using ground plane aperture technique," IEE Proceedings: Microwaves, Antennas and Propagation, vol. 149, no. 1, pp. 71-77, 2002.

[12] Z. Y. Zhang, Y.-X. Guo, L. C. Ong, and M. Y. W. Chia, "Analysis of parallel coupled line bandpass filters using a ground-plane aperture," Microwave and Optical Technology Letters, vol. 45, no. 3, pp. 237-240, 2005.

[13] F. Masot, F. Medina, and M. Horno, "Theoretical and experimental study of modified coupled strip coupler," Electronics Letters, vol. 28, no. 4, pp. 347-348, 1992.

[14] A. M. Abbosh, "Broadband quadrature coupler with slotted ground plane," Microwave and Optical Technology Letters, vol. 50, no. 2, pp. 328-331, 2008.

[15] M. C. Velázquez-Ahumada, J. Martel, and F. Medina, "Parallel coupled microstrip filters with ground-plane aperture for spurious band suppression and enhanced coupling," IEEE Transactions on Microwave Theory and Techniques, vol. 52, no. 3, pp. 1082-1086, 2004.

[16] M. C. Velázquez-Ahumada, J. Martel, and F. Medina, "Parallel coupled microstrip filters with floating ground-plane conductor for spurious-band suppression," IEEE Transactions on Microwave Theory and Techniques, vol. 53, no. 5, pp. 1823-1828, 2005.

[17] Y.-X. Guo, Z.-Y. Zhang, and L. C. Ong, "Improved wideband Schiffman phase shifter," IEEE Transactions on Microwave Theory and Techniques, vol. 54, no. 3, pp. 1196-1200, 2006.

[18] A. M. Abbosh, "Broadband parallel-coupled quadrature coupler with floating-potential ground plane conductor," Microwave and Optical Technology Letters, vol. 50, no. 9, pp. 2304-2307, 2008.

[19] A. M. Abbosh, "Analytical closed-form solutions for different configurations of parallel-coupled microstrip lines," IET Microwaves, Antennas and Propagation, vol. 3, no. 1, pp. 137-147, 2009.

[20] F. Güneş and N. Türker, "Artificial neural networks in their simplest forms for analysis and synthesis of $\mathrm{RF} /$ microwave 
planar transmission lines," International Journal of $R F$ and Microwave Computer-Aided Engineering, vol. 15, no. 6, pp. 587600, 2005.

[21] C. Yildiz, K. Guney, M. Turkmen, and S. Kaya, "Neural models for coplanar strip line synthesis," Progress in Electromagnetics Research, vol. 69, pp. 127-144, 2007.

[22] Z. Wang, S. Fang, and S. Fu, "ANN synthesis models trained with modified GA-LM algorithm for ACPWs with conductor backing and substrate overlaying," ETRI Journal, vol. 34, no. 5, pp. 696-705, 2012.

[23] K. A. Alshamaileh, M. J. Almalkawi, R. R. Junuthula, V. K. Devabhaktuni, and P. H. Aaen, "ANN-based modeling of compact impedance-varying transmission lines with applications to ultra-wideband Wilkinson power dividers," International Journal of RF and Microwave Computer-Aided Engineering, vol. 25, no. 7, pp. 563-572, 2015.

[24] S. Haykin, Neural Networks: A Comprehensive Foundation, Prentice-Hall, Upper Saddle River, NJ, USA, 2nd edition, 1998.

[25] Q. J. Zhang and K. C. Gupta, Neural Networks for RF and Microwave Design, Artech House, Norwood, Mass, USA, 2000.

[26] H. Kabir, L. Zhang, M. Yu, P. H. Aaen, J. Wood, and Q.-J. Zhang, "Smart modeling of microwave devices," IEEE Microwave Magazine, vol. 11, no. 3, pp. 105-118, 2010.

[27] Z. Wang and S. Fang, "ANN synthesis model of single-feed corner-truncated circularly polarized microstrip antenna with an air gap for wideband applications," International Journal of Antennas and Propagation, vol. 2014, Article ID 392843, 7 pages, 2014.

[28] Z. Wang, S. Fang, Q. Wang, and H. Liu, "An ANN-based synthesis model for the single-feed circularly-polarized square microstrip antenna with truncated corners," IEEE Transactions on Antennas and Propagation, vol. 60, no. 12, pp. 5989-5992, 2012. 


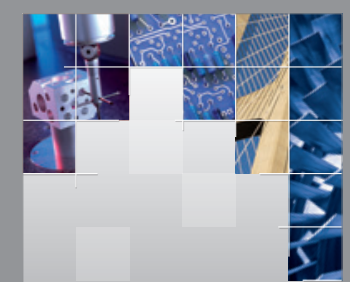

\section{Enfincering}
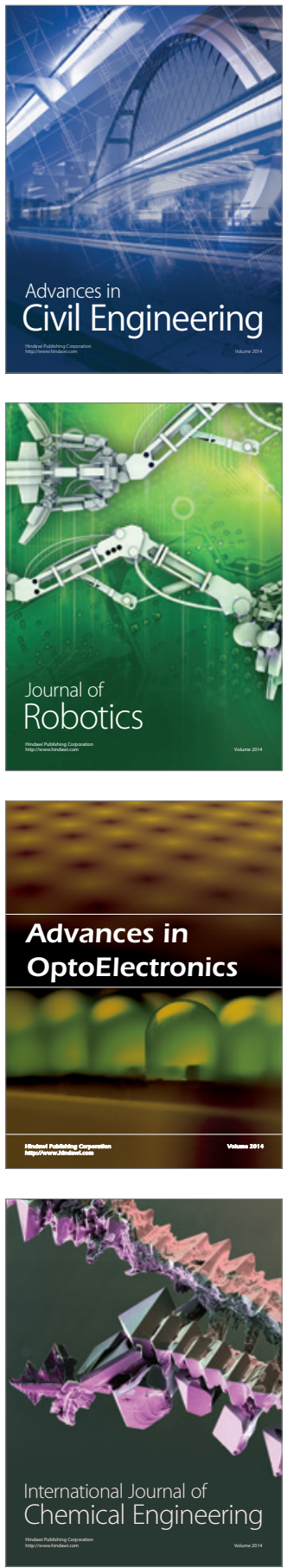

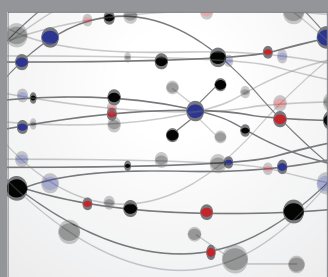

The Scientific World Journal

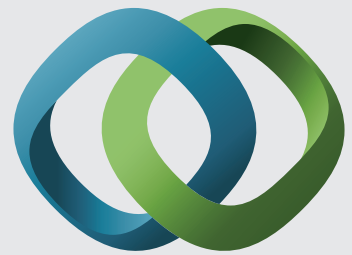

\section{Hindawi}

Submit your manuscripts at

http://www.hindawi.com
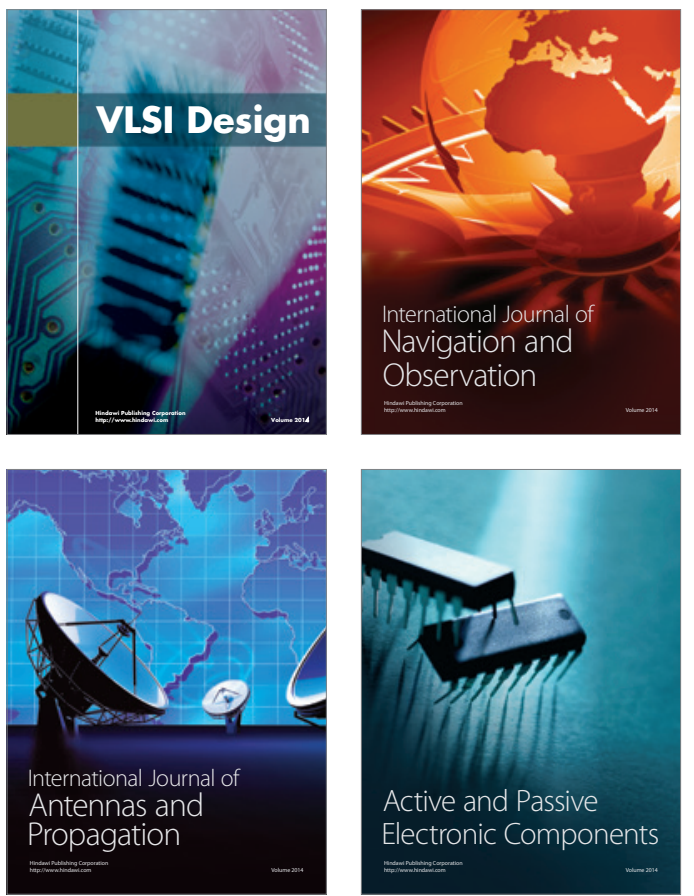
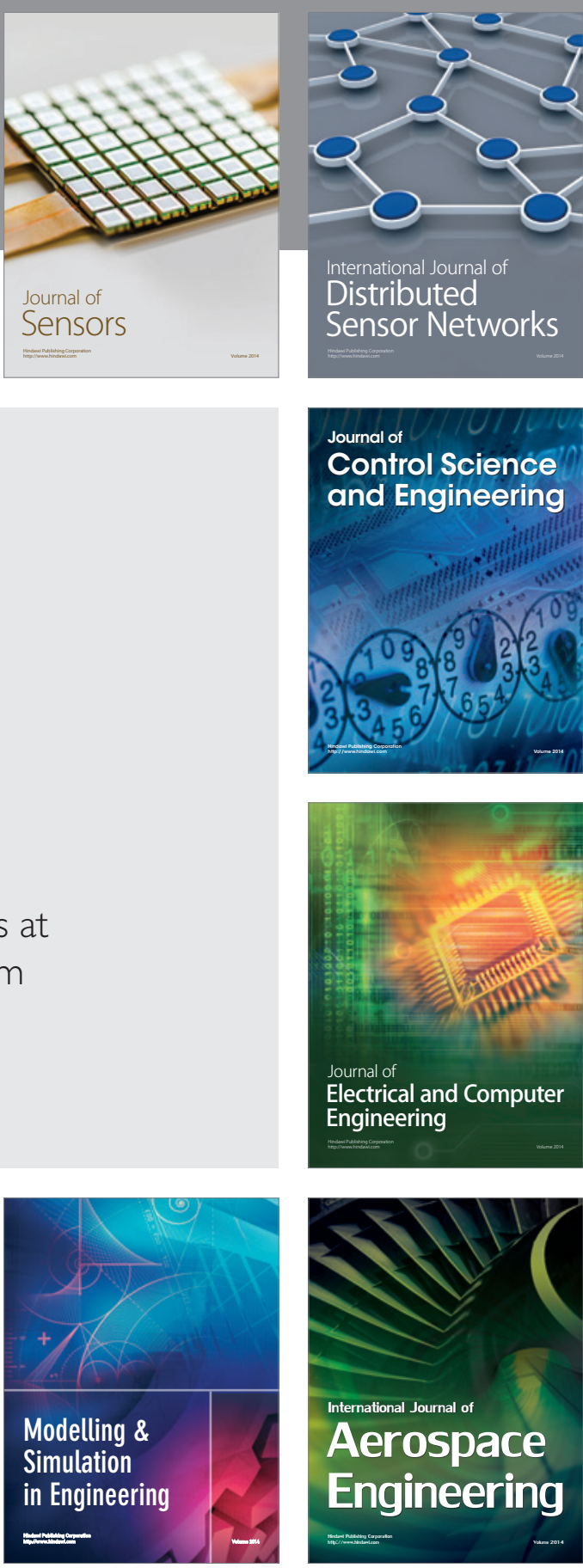

International Journal of

Distributed

Sensor Networks

Journal of

Control Science

and Engineering
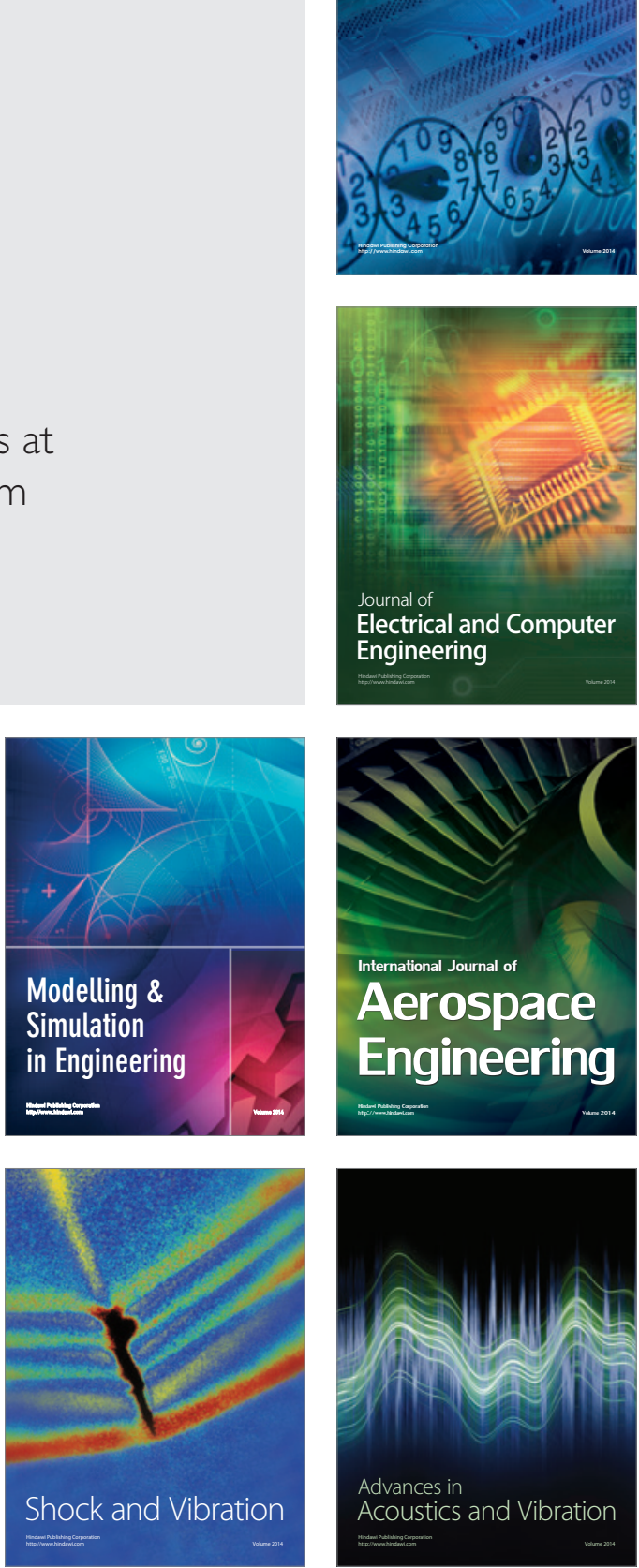